\title{
Posttranslational Regulation of the NLR Family Pyrin Domain-Containing 3 Inflammasome
}

\author{
Do-Wan Shim ${ }^{1}$ and Kwang-Ho Lee ${ }^{1,2 *}$ \\ 'Department of Applied Life Science, Graduate School, Konkuk University, Chungju, South Korea, ${ }^{2}$ Department of \\ Biotechnology, College of Biomedical and Health Science, Research Institute of Inflammatory Disease, \\ Konkuk University, Chungju, South Korea
}

OPEN ACCESS

Edited by:

Charles Dinarello,

University of Colorado Denver,

United States

Reviewed by:

Alessandra Mortellaro,

Istituto San Raffaele

Telethon per la Terapia

Genica (SR-Tiget), Italy

Carlos De Torre,

IMIB-Arrixaca, Spain

*Correspondence:

Kwang-Ho Lee

kwangho@kku.ac.kr

Specialty section: This article was submitted

to Inflammation,

a section of the journal

Frontiers in Immunology

Received: 10 February 2018

Accepted: 27 April 2018

Published: 18 May 2018

Citation:

Shim D-W and Lee K-H (2018) Posttranslational

Regulation of the NLR

Family Pyrin Domain-

Containing 3 Inflammasome.

Front. Immunol. 9:1054. doi: 10.3389/fimmu.2018.01054
The NOD-like receptor family pyrin domain-containing 3 (NLRP3) inflammasome is a multi-protein complex that can be activated by a variety of pathogen-associated molecular patterns or damage-associated molecular patterns. Inappropriate NLRP3 inflammasome activation can induce autoinflammatory, autoimmune, or metabolic disorders. Therefore, NLRP3 is an attractive target against NLRP3 inflammasome activation, and specific targeting of NLRP3 might be an intriguing approach to the development of drugs for the treatment of NLRP3 inflammasome-related diseases. Although many studies with varied mechanistic approaches were reported in inhibition of NLRP3 inflammasome activation, mechanisms related to regulation of posttranslational modification (PTM) of NLRP3, as a focal point has not been thoroughly addressed. Recently, extensive investigations of PTMs of NLRP3 have led to partial understanding of the mechanisms involved in NLRP3 inflammasome activation. In this review, we focused on the role of PTMs regulating NLRP3 inflammasome activation.

Keywords: NLR family pyrin domain-containing 3, inflammasome, posttranslational regulation, ubiquitination, phosphorylation, alkylation, S-nitrosylation

\section{INTRODUCTION}

Inflammasomes are unique, complex structures formed by proteins in cytosol. Inflammasome activation results in the recruitment and activation of caspase-1, which then cleaves pro-IL-1 $\beta$ and pro-IL-18 into their active forms. Inflammasomes are characterized by a particular sensor or receptor molecule, such as absent in melanoma 2 (AIM2), IFN $\gamma$-inducible protein 16 (IFI16), and various Nod-like receptor (NLR) subsets $(1,2)$. Among a number of inflammasomes, the NLR family pyrin domain (PYD)-containing 3 (NLRP3) inflammasome is the most well studied, because it responds to infectious pathogens, such as Sendai virus, adenovirus, influenza virus (3), Escherichia coli, Staphylococcus aureus (4), and host-derived damage-associated molecular patterns, including extracellular adenosine triphosphate (ATP) (5), monosodium urate (MSU) crystals (6), cholesterol (7), silica (8), aluminum salts (9), amyloid deposits (10), and fatty acids (11).

The autoinflammatory syndromes associated with gain-of-function mutations of NLRP3 leads to abnormal NLRP3 inflammasome activation causing cryopyrin-associated periodic syndromes (CAPS), a group of rare, inherited, auto-inflammatory diseases, such as Muckle-Wells syndrome, familial cold urticaria, and neonatal onset multisystem inflammatory disease (NO-MID) $(12,13)$. Reports also revealed that excessive activation of NLRP3 inflammasome could play an important role in other diseases, such as multiple sclerosis (14), Alzheimer's disease (15), metabolic disorders, such as gout (6), atherosclerosis (16) and type 2 diabetes (11, 17-19). NLRP3 comprises a PYD, 
a nucleotide-binding domain (NBD), and leucine-rich repeat (LRR) motif (20). The NLRP3 inflammasome is a multi-protein complex mainly composed of NLRP3, ASC, and caspase-1 (21). Therefore, NLRP3 is an ideal target for the development of specific inhibitors against the NLRP3 inflammasome.

There have been many efforts to develop inhibitors specific to the NLRP3 inflammasome by targeting NLRP3. Strategies directing toward posttranslationally modified NLRP3 are particularly intriguing methods in developing NLRP3-specific inhibitors.

Posttranslational modifications (PTMs), which are carried out by covalent bonding of low-molecular weight groups, such as alkyl groups, phosphate groups, or the ubiquitin protein, to amino acids, are closely related to numerous physiological activities of proteins. PTMs were also known to control immune responses through the regulation of protein folding, location, stability, and interaction with other molecules $(22,23)$. Many types of PTMs, such as phosphorylation, ubiquitination, alkylation, and S-nitrosylation have been detected in NLRP3, and these PTMs play important roles in NLRP3 inflammasome activation (24). In this review, we focus on how individual PTMs are involved in NLRP3 and regulate NLRP3 inflammasome activation and its subsequent functional roles.

\section{NLRP3: PHOSPHORYLATION}

Phosphorylation of NLRP3 was first reported by Spalinger et al. (25), and so far, five different phosphorylation sites have been proposed (25-30).

\section{Protein Tyrosine Phosphatase Non-Receptor 22 (PTPN22)}

The PTPN22, a protein tyrosine phosphatase, is known to be associated with several inflammatory disorders, including
Crohn's disease (31), rheumatoid arthritis (32), systemic lupus erythematosus (33), and type-1 diabetes (34). Recently, Spalinger et al. reported that PTPN22 positively regulates NLRP3 inflammasome activation through its phosphatase activity, but does not similarly regulate AIM2 and NLRC4 inflammasome activation (25). Phosphorylation of the Y861 residue at the LRR domain of NLRP3 negatively regulates NLRP3 inflammasome activation, but NLRP3 inflammasome stimulators, such as ATP, MSU crystals, and silica crystals, induce tyrosine dephosphorylation of NLRP3 at Y861 in a PTPN22-dependent manner, leading to activation of NLRP3 (25). PTPN22 directly interacts with NLRP3 in NLRP3 inflammasome-activated macrophages (25). PTPN22 deficiency prevents MSU-induced peritonitis in mice by inhibition of inflammasome-dependent IL- $1 \beta$ production (25). These previous reports are summarized in Table $\mathbf{1}$ and Figure $\mathbf{1}$. However, kinases related to NLRP3 tyrosine phosphorylation remain to be identified.

\section{Protein Kinase A (PKA)}

Protein kinase A is a serine/threonine kinase important in controlling the immune system by modulating gene transcription and post-translational signaling (35). Recently, Sokolowska et al. suggested that PKA inhibits NLRP3 inflammasome activation through the induction of NLRP3 serine phosphorylation (36). Prostaglandin E2 (PGE2) stimulates the E-prostanoid 4 (EP4) receptor and activates PKA signaling by increasing cytoplasmic cyclic adenosine monophosphate (cAMP) levels (36). PGE2mediated EP4 signaling causes PKA-NLRP3 interactions, and subsequently activates PKA, then phosphorylates NLRP3 within the NBD at S295 by increasing cytoplasmic cAMP levels (Table 1; Figure 1). Phosphorylation of the S295 residue in NLRP3 negatively regulates NLRP3 inflammasome activation through reduction of the ATPase activity of NLRP3 (26). Bile acids also

TABLE 1 | Regulation of NLR family pyrin domain (PYD)-containing 3 (NLRP3) inflammasome activation through posttranslational modification (PTM) of NLRP3.

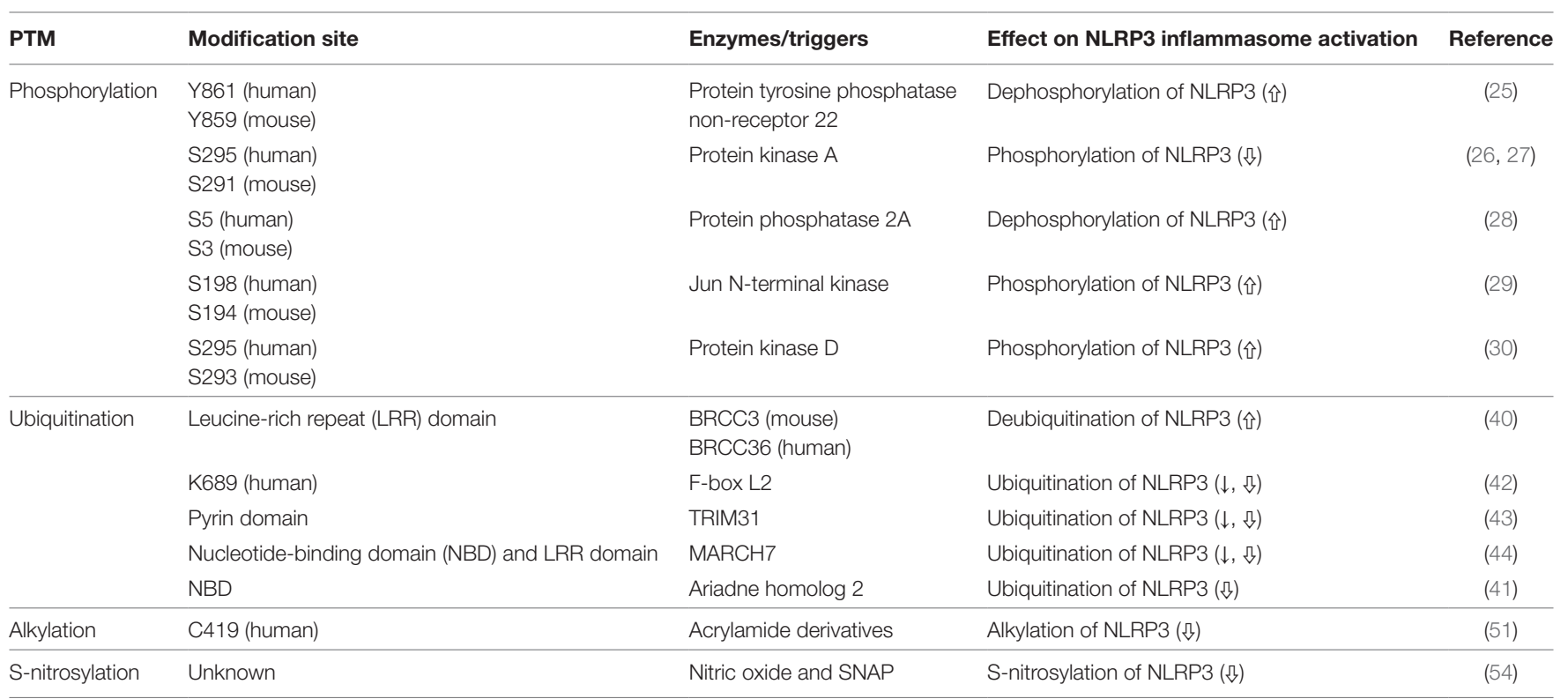

$\downarrow$, Decrease of NLRP3 protein level; 仓, activation of NLRP3 inflammasome; 凤, inhibition of NLRP3 inflammasome. 


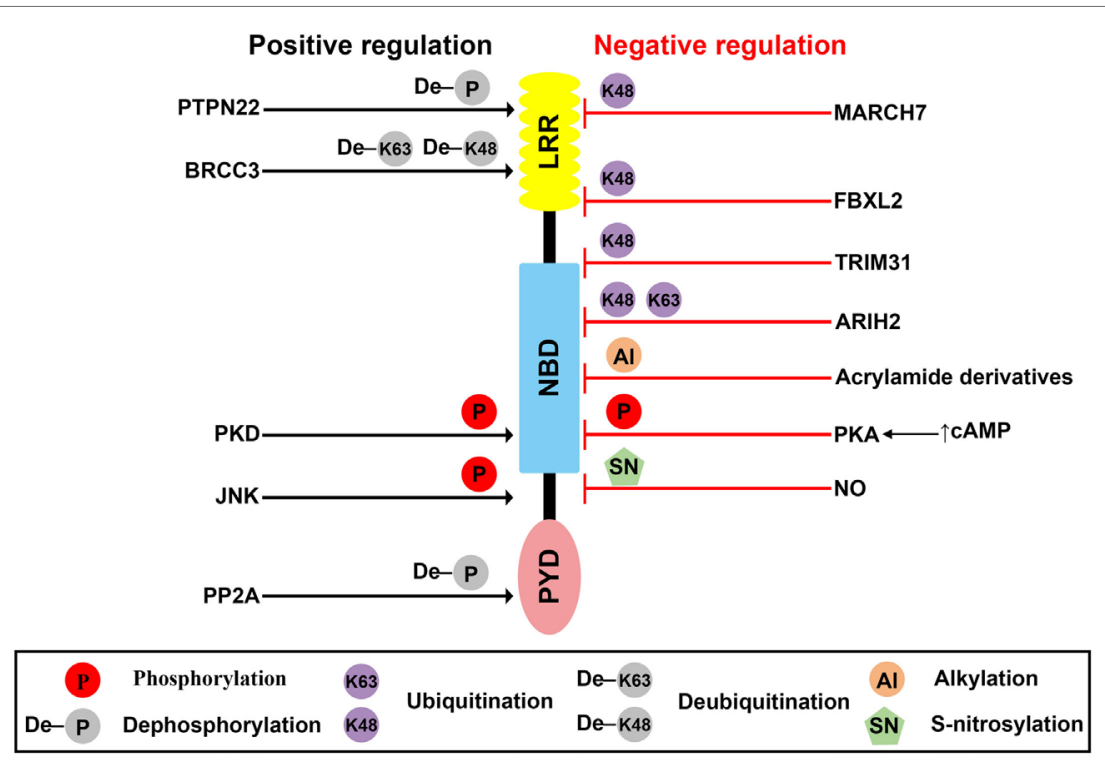

FIGURE 1 | Posttranslational modification (PTMs) of NLR family pyrin domain (PYD)-containing 3 (NLRP3). Schematic of NLRP3 structure and mechanisms of posttranslational regulation of the NLRP3 inflammasome are represented. PTMs that promote activation of the inflammasome are shown as black arrows, whereas those that suppress inflammasome activation are shown as red blocks.

phosphorylate the 295 residue in NLRP3 through the cAMP-PKA axis (27). Bile acids stimulate transmembrane $G$ protein-coupled receptor-5 (TGR5), TGR5 signaling activates PKA through increasing cytoplasmic cAMP levels, and activated PKA further induces phosphorylation of the S295 residue in NLRP3, leading to NLRP3 inhibition. Bile acid and TGR5 signaling promotes mixed K63 and K48 ubiquitination of NLRP3 through phosphorylation of the S295 residue in NLRP3 (27). Forskolin, a PKA activator that increases cytoplasmic cAMP levels inhibits NLRP3 inflammasome activation by phosphorylation of the S295 residue in NLRP3. Several CAPS mutations in the region between residues I290 and D311 show that phosphorylation of the S295 residue in NLRP3 are impaired in CAPS-mutant NLRP3 (26). In an in vivo experiment, PKA activation attenuated both LPS-induced sepsis and alum-induced peritonitis via suppression of NLRP3 inflammasome activation. Further, PKA activation improved insulin sensitivity in high-fat diet-induced type-2 diabetes through suppression of NLRP3 inflammasome activation (27).

\section{Protein Phosphatase 2A (PP2A)}

Phosphorylation of the S5 residue in the PYD of NLRP3 negatively regulates NLRP3 inflammasome activation. Phosphorylation of the S5 residue in NLRP3 causes electrostatic repulsion of PYDs. PP2A promotes NLRP3 inflammasome activation through dephosphorylation of the S5 residue in NLRP3 (Table 1; Figure 1). Okadaic acid, a PP2A inhibitor, significantly attenuates NLRP3 inflammasome activation. NLRP3 inflammasome activation is impaired in PP2A-deficient macrophages (28). However, Martin et al. suggested that PP2A is also related to localization of ASC through the regulation of ASC phosphorylation via decreasing IKK $\alpha$ kinase activity in a PP2A-dependent manner (37). According to their study, PP2A induced dephosphorylation of IKK $\alpha$ through the interaction with IKK $\alpha$ in NLRP3 inflammasome-activated macrophages (37). For this reason, the specificity of PP2A is not yet clear, and the kinase involved in the phosphorylation of the S5 residue in NLRP3 remains to be identified.

\section{Jun N-Terminal Kinase (JNK)}

C-Jun N-terminal kinase-mediated NLRP3 phosphorylation is an essential priming event for NLRP3 inflammasome activation. NLRP3 is directly phosphorylated by JNK1 during priming, but not by JNK2. All toll-like receptor ligands phosphorylate the S194 residue in NLRP3 in a JNK1-dependent manner (Table 1; Figure 1). JNK1-mediated NLRP3 phosphorylation promotes the deubiquitination of NLRP3 and facilitates the self-association of NLRP3. NLRP3 inflammasome activation is completely inhibited in JNK1-deficient macrophages, and JNK inhibitors also completely inhibit NLRP3 inflammasome activation, but do not inhibit AIM2 or NLRC4 inflammasome activation. NLRP3 S194A mutants fail to activate the NLRP3 inflammasome both in vitro and in vivo. S194A-mutant NLRP3 expressed in mice prevented both MSU-induced peritonitis and LPS-induced sepsis through inhibition of NLRP3 inflammasome activation (29).

\section{Protein Kinase D}

Protein kinase D (PKD)-mediated NLRP3 phosphorylation is required for full activation of the NLRP3 inflammasome. During the NLRP3 inflammasome activation process, NLRP3 directly binds to mitochondria-associated membranes (MAMs) and NLRP3 protein level increases in MAMs fraction $(38,39)$. Zhang et al. suggested that during NLRP3 inflammasome activation process, MAMs localized to adjacent Golgi membrane and diacylglycerol (DAG) increased at the Golgi (30). As DAG accumulation at 
Golgi occurred at upstream of NLRP3 inflammasome activation, deletion of NLRP3 could not affect the DAG accumulation at Golgi. DAG accumulation at Golgi triggers the activation of PKD, a key effector of DAG activation. Activated PKD subsequently interacts with NBD of NLRP3 and phosphorylates the S293 residue in NLRP3. PKD-mediated NLRP3 phosphorylation leads to release of NLRP3 from MAMs to cytoplasm, allowing full NLRP3 inflammasome maturation. Both inhibition of PKD activity and PKD-deficient macrophages resulted in decrease of NLRP3 inflammasome activation which is due to the inhibition of NLRP3 release from MAMs to cytoplasm. PKD inhibition prevents NLRP3 inflammasome activation in peripheral blood mononuclear cells from CAPS patients. PKD inhibitor also prevents both LPS-induced systemic inflammation and S. aureusinduced inflammation in in vivo through the inhibition of NLRP3 inflammasome activation (30).

\section{NLRP3: UBIQUITINATION}

NLR family pyrin domain (PYD)-containing 3 ubiquitination was first reported by Py et al. (40), and two different kinds of NLRP3 ubiquitination-mediated negative regulation of NLRP3 have been reported: mixed K63 and K48 ubiquitination-mediated NLRP3 inactivation $(40,41)$ and K48 ubiquitination-mediated proteasomal degradation of NLRP3 (42-44). The drugs that increase ubiquitinated-NLRP3 population, such as G5 were widely used to inhibit NLRP3 inflammasome activation (40).

\section{BRCC3}

Deubiquitination of NLRP3 is essential to NLRP3 inflammasome activation, and some deubiquitinase inhibitors negatively regulate NLRP3 inflammasome activation. The priming signal of LPS stimulation is important not only for increasing expression levels of NLRP3 inflammasome components, such as NLRP3 and pro-IL-1 $\beta$, but also for inducing NLRP3 deubiquitination, which puts NLRP3 in its ready state $(40,45)$. Py et al. suggested that the deubiquitinase BRCC3 played an important role in NLRP3 inflammasome activation through deubiquitination of NLRP3 and interaction with the LRR domain of NLRP3 (Table 1; Figure 1). Inhibition of NLRP3 deubiquitination by G5, a BRCC3 inhibitor, increased the polyubiquitination of NLRP3 with mixed K63 and K48 chains, which critically inhibited NLRP3 inflammasome activation, and this ubiquitination was not related to protein degradation (40). During the NLRP3 inflammasome activation process, both the priming and activation signal can induce deubiquitination of NLRP3, and these two independent deubiquitination signals are important to NLRP3 inflammasome activation. Priming signal-mediated NLRP3 deubiquitination is induced in a reactive oxygen species-dependent manner, but the mechanism activating signal-mediated NLRP3 deubiquitination is unknown (46). A recent study suggested that kinase activity of JNK, which is a representative stress signal, was essential to priming signal-mediated NLRP3 deubiquitination through induction of NLRP3 phosphorylation (29). However, the exact ubiquitination site, ubiquitin E3 ligase, and mechanism activating signal-mediated deubiquitination of NLRP3 remain to be identified.

\section{F-Box L2 (FBXL2)}

Protein degradation-induced ubiquitination has also been detected in NLRP3. A recent study suggested that the Skp-CullinF-box (SCF) family member, FBXL2, which is a ubiquitin E3 ligase, directly interacted with NLRP3, leading to proteasomal degradation of NLRP3 through ubiquitination. FBXL2 recognizes and interacts with the tryptophan-73 residue within NLRP3, and then subsequently ubiquitinates the lysine-689 residue, leading to NLRP3 degradation (Table 1; Figure 1). FBXL2 regulates stability of the NLRP3 protein through ubiquitination-mediated proteasomal degradation. The LPS priming signal increases stability of the NLRP3 protein through reducing FBXL2 levels. LPS activates F-box $\mathrm{O} 3$ (FBXO3), and the activated $\mathrm{FBXO} 3$ ubiquitinates and degrades FBXL2. BC-1215, an FBXO3 inhibitor, significantly inhibits NLRP3 inflammasome activation by decreasing NLRP3 expression levels via increasing FBXL2 expression levels (42). The LPS priming signal is important not only to increase NLRP3 protein levels but also to extend the half-life of the NLRP3 protein, creating a suitable environment for NLRP3 inflammasome activation. However, whether BRCC3 can deubiquitinate the K689 residue in NLRP3 through FBXL2 is unclear.

\section{TRIM31}

TRIM31, an E3 ubiquitin ligase, also causes K48-linked ubiquitination of NLRP3, leading to its proteasomal degradation and thus reducing inflammasome activation. TRIM31 directly interacts with the PYD of NLRP3 (Table 1; Figure 1). Intracellular TRIM31 expression levels increase by stimulation with either LPS or IL-1 $\beta$ in macrophages, suggesting that TRIM31 is a part of feedback suppressor of the NLRP3 inflammasome through reduction in NLRP3 expression. In an in vivo experiment, TRIM31-deficient mice exhibited higher concentrations of IL- $1 \beta$ in the serum, but not of TNF- $\alpha$ or IL- 6 in an LPS-induced peritonitis model. The mice also showed increased recruitment of neutrophils and monocytes to the peritoneum in alum-induced peritonitis through hyperactivation of the NLRP3 inflammasome. These data suggested that TRIM31-mediated ubiquitination and degradation of NLRP3 negatively regulated NLRP3 inflammasome activation (43).

\section{MARCH7}

Dopamine, which functions as a neurotransmitter in the brain, inhibits NLRP3 inflammasome activation through MARCH7mediated ubiquitination and autophagic degradation of NLRP3 in response to stimulation of the dopamine D1 receptor(DRD1) (44). Although the dopamine concentration (approximately $100 \mu \mathrm{M}$ ) required for NLRP3 inflammasome inhibition in vitro is higher than the reported physiological concentration of dopamine (approximately $1 \mu \mathrm{M})(47,48)$, dopamine-mediated DRD1 signaling induced degradation of NLRP3, but not other inflammasome components, and this degradation was prevented by autophagic inhibition, but not by inhibition of proteasomal degradation. DRD1 signaling increases cytoplasmic cAMP levels, which in turn cause not only MARCH7-dependent K48-linked ubiquitination but also autophagic degradation of NLRP3. MARCH7 reportedly binds both the NBD and LRR domains of NLRP3 (Table 1; Figure 1). Dopamine does not inhibit NLRP3 inflammasome activation in MARCH7-deficient macrophages. 
Dopamine-mediated DRD1 signaling prevents MPTP-induced neurodegeneration through suppression of the NLRP3 inflammasome. Dopamine also prevents MPTP-induced dopaminergic neuronal cell death and reduced NLRP3 expression levels. However, dopamine does not prevent MPTP-induced neurodegenerative disease in DRD1-deficient mice (44). Dopaminemediated DRD1 signaling also prevents both LPS-induced systemic inflammation and MSU-induced peritonitis through suppression of IL-1 $\beta$ production via inhibition of NLRP3 inflammasome activation, but not by suppression of TNF- $\alpha$ (44).

\section{Ariadne Homolog 2 (ARIH2)}

Ariadne homolog 2, an E3 ubiquitin ligase, negatively regulates NLRP3 inflammasome activation by inducing mixed K63 and K48 ubiquitination of NLRP3. The RING2 domain of ARIH2 is critical for the interaction with the NBD of NLRP3 (Table 1; Figure 1). Further, ARIH2 is co-localized with NLRP3 inflammasome components, such as NLRP3 and ASC, in NLRP3 inflammasome-activated macrophages. ARIH2-deficient macrophages exhibit increased NLRP3 inflammasome activation through reduction of NLRP3 ubiquitination. On the contrary, ARIH2-overexpressing macrophages prevent NLRP3 inflammasome activation by increasing NLRP3 ubiquitination. Thus, ARIH2 is an endogenous posttranslational negative regulator of NLRP3 inflammasome activation in macrophages (41).

\section{NLRP3: ALKYLATION}

NLR family pyrin domain (PYD)-containing 3 alkylation was first reported by Juliana et al. (49), and some NLRP3 inflammasome-specific inhibitors, including Bay11-7082, 3,4-methylenedioxy- $\beta$-nitrostyrene (MNS), some acrylamide derivatives, and 2-cyclohexylimino-6-methyl-6,7-dihydro$5 \mathrm{H}$-benzo[1,3] oxathiol-4-one (BOT-4-one) work via direct alkylation of NLRP3. These chemicals commonly have an electrophilic region, and this region forms covalent bonds with NLRP3 (49-51). Cocco et al. predicted the Cys419 residue in the ATPase catalytic pocket of NLRP3 as the plausible alkylation site for acrylamide derivatives via computational analysis (Table 1; Figure 1) (51). The functional role of NLRP3-alkylating agents commonly appears to be critically mediated by inhibition of NLRP3 ATPase activity. NLRP3 alkylation reduces the ATPbinding affinity of NLRP3 (49), thereby resulting in impaired ATPase activity $(50,51)$. The ATPase activity of NLRP3 is important both to NLRP3 self-association and to NLRP3-ASC

\section{REFERENCES}

1. Franchi L, Eigenbrod T, Muñoz-Planillo R, Nuñez G. The inflammasome: a caspase-1-activation platform that regulates immune responses and disease pathogenesis. Nat Immunol (2009) 10:241-7. doi:10.1038/ni.1703

2. Franchi L, Muñoz-Planillo R, Núñez G. Sensing and reacting to microbes through the inflammasomes. Nat Immunol (2012) 13:325-32. doi:10.1038/ ni. 2231

3. Gram AM, Frenkel J, Ressing ME. Inflammasomes and viruses: cellular defence versus viral offence. J Gen Virol (2012) 93:2063-75. doi:10.1099/vir.0.042978-0

4. Rathinam VA, Vanaja SK, Waggoner L, Sokolovska A, Becker C, Stuart LM, et al. TRIF licenses caspase-11-dependent NLRP3 inflammasome activation association (52). Recently, Shim et al. suggested that NLRP3 alkylation is also related to ubiquitination of NLRP3. NLRP3alkylating agents, such as Bay11-7082, MNS, and BOT-4-one, increase ubiquitinated NLRP3 levels through their alkylating activity (53). Some electrophilic chemicals that mediate NLRP3 alkylation negatively regulate NLRP3 inflammasome activation, but the exact alkylation site in NLRP3 should be identified by crystallization and mass spectrometry.

\section{NLRP3: S-NITROSYLATION}

Nitric oxide (NO) negatively regulates NLRP3 inflammasome activation through S-nitrosylation of NLRP3. NO is important to the inhibition of bacterial growth and can be induced by $\mathrm{T}$ cell-derived interferon- $\gamma($ IFN- $\gamma$ ) in macrophages. Both IFN$\gamma$-induced NO production and NO donor (SNAP) treatment inhibit canonical and Mycobacterium tuberculosis-mediated NLRP3 inflammasome activation through induction of NO-induced S-nitrosylation of NLRP3 (Table 1; Figure 1) (54). However, the exact S-nitrosylation site of NLRP3 remains to be identified.

\section{PERSPECTIVE}

Here we focused on the role of PTMs of NLRP3 in NLRP3 inflammasome activation. NLRP3 is clearly subjected to various PTMs involved in the precise activation of the NLRP3 inflammasome. PTMs of NLRP3 may be good targets for the development of NLRP3-specific drugs or inhibitors. However, the exact PTM sites of NLRP3 and PTM-related enzymes remain to be identified, and the correlations and interactions among various types of PTMs of NLRP3 warrant future investigation.

\section{AUTHOR CONTRIBUTIONS}

D-WS drafted the manuscript, figure, and table. K-HL supervised and edited the manuscript, figure, and table.

\section{ACKNOWLEDGMENTS}

We are grateful to Dr. S. Koppula and Dr. TB Kang for their helpful comments. This research was supported by Basic Science Research Program through the National Research Foundation of Korea (NRF) funded by the Ministry of Education (2016-A4190098).

by Gram-negative bacteria. Cell (2012) 150:606-19. doi:10.1016/j.cell.2012. 07.007

5. Mariathasan S, Weiss DS, Newton K, McBride J, O’rourke K, Roose-Girma M, et al. Cryopyrin activates the inflammasome in response to toxins and ATP. Nature (2006) 440:228. doi:10.1038/nature04515

6. Martinon F, Pétrilli V, Mayor A, Tardivel A, Tschopp J. Gout-associated uric acid crystals activate the NALP3 inflammasome. Nature (2006) 440:237. doi:10.1038/nature04516

7. Duewell P, Kono H, Rayner KJ, Sirois CM, Vladimer G, Bauernfeind FG, et al. NLRP3 inflammasomes are required for atherogenesis and activated by cholesterol crystals. Nature (2010) 464:1357-61. doi:10.1038/ nature 08938 
8. Dostert C, Petrilli V, Van Bruggen R, Steele C, Mossman BT, Tschopp J. Innate immune activation through Nalp3 inflammasome sensing of asbestos and silica. Science (2008) 320:674-7. doi:10.1126/science.1156995

9. Eisenbarth SC, Colegio OR, O'Connor W, Sutterwala FS, Flavell RA. Crucial role for the Nalp3 inflammasome in the immunostimulatory properties of aluminium adjuvants. Nature (2008) 453:1122-6. doi:10.1038/ nature 06939

10. Halle A, Hornung V, Petzold GC, Stewart CR, Monks BG, Reinheckel T, et al. The NALP3 inflammasome is involved in the innate immune response to amyloid- $\beta$. Nat Immunol (2008) 9:857-65. doi:10.1038/ni.1636

11. Wen H, Gris D, Lei Y, Jha S, Zhang L, Huang MT, et al. Fatty acid-induced NLRP3-ASC inflammasome activation interferes with insulin signaling. Nat Immunol (2011) 12:408-15. doi:10.1038/ni.2022

12. Hoffman HM, Brydges SD. Genetic and molecular basis of inflammasomemediated disease. J Biol Chem (2011) 286:10889-96. doi:10.1074/jbc.R110. 135491

13. Lamkanfi M, Dixit VM. Inflammasomes: guardians of cytosolic sanctity. Immunol Rev (2009) 227:95-105. doi:10.1111/j.1600-065X.2008.00730.x

14. Gris D, Ye Z, Iocca HA, Wen H, Craven RR, Gris P, et al. NLRP3 plays a critical role in the development of experimental autoimmune encephalomyelitis by mediating Th1 and Th17 responses. J Immunol (2010) 185:974-81. doi:10.4049/jimmunol.0904145

15. Heneka MT, Kummer MP, Stutz A, Delekate A, Schwartz S, Vieira-Saecker A, et al. NLRP3 is activated in Alzheimer's disease and contributes to pathology in APP/PS1 mice. Nature (2013) 493:674-8. doi:10.1038/nature11729

16. Walle LV, Van Opdenbosch N, Jacques P, Fossoul A, Verheugen E, Vogel P, et al. Negative regulation of the NLRP3 inflammasome by A20 protects against arthritis. Nature (2014) 512:69-73. doi:10.1038/nature13322

17. Stienstra R, Joosten LA, Koenen T, Van Tits B, Van Diepen JA, van den Berg SA, et al. The inflammasome-mediated caspase-1 activation controls adipocyte differentiation and insulin sensitivity. Cell Metab (2010) 12:593-605. doi:10.1016/j.cmet.2010.11.011

18. Vandanmagsar B, Youm Y, Ravussin A, Galgani JE, Stadler K, Mynatt RL, et al. The NLRP3 inflammasome instigates obesity-induced inflammation and insulin resistance. Nat Med (2011) 17:179-88. doi:10.1038/ nm.2279

19. Stienstra R, van Diepen JA, Tack CJ, Zaki MH, van de Veerdonk FL, Perera D, et al. Inflammasome is a central player in the induction of obesity and insulin resistance. Proc Natl Acad Sci U S A (2011) 108:15324-9. doi:10.1073/pnas.1100255108

20. Ting JP, Lovering RC, Alnemri ES, Bertin J, Boss JM, Davis BK, et al. The NLR gene family: a standard nomenclature. Immunity (2008) 28:285-7. doi:10.1016/j.immuni.2008.02.005

21. Guo H, Callaway JB, Ting JP. Inflammasomes: mechanism of action, role in disease, and therapeutics. Nat Med (2015) 21:677-87. doi:10.1038/ nm.3893

22. Liu J, Qian C, Cao X. Post-translational modification control of innate immunity. Immunity (2016) 45:15-30. doi:10.1016/j.immuni.2016.06.020

23. Deribe YL, Pawson T, Dikic I. Post-translational modifications in signal integration. Nat Struct Mol Biol (2010) 17:666-72. doi:10.1038/nsmb.1842

24. Baker PJ, De Nardo D, Moghaddas F, Tran LS, Bachem A, Nguyen T, et al. Posttranslational modification as a critical determinant of cytoplasmic innate immune recognition. Physiol Rev (2017) 97:1165-209. doi:10.1152/ physrev.00026.2016

25. Spalinger MR, Kasper S, Gottier C, Lang S, Atrott K, Vavricka SR, et al. NLRP3 tyrosine phosphorylation is controlled by protein tyrosine phosphatase PTPN22. J Clin Invest (2016) 126:1783-800. doi:10.1172/JCI83669

26. Mortimer L, Moreau F, MacDonald JA, Chadee K. NLRP3 inflammasome inhibition is disrupted in a group of auto-inflammatory disease CAPS mutations. Nat Immunol (2016) 17:1176-86. doi:10.1038/ni.3538

27. Guo C, Xie S, Chi Z, Zhang J, Liu Y, Zhang L, et al. Bile acids control inflammation and metabolic disorder through inhibition of NLRP3 inflammasome. Immunity (2016) 45:802-16. doi:10.1016/j.immuni.2016.09.008

28. Stutz A, Kolbe CC, Stahl R, Horvath GL, Franklin BS, van Ray O, et al. NLRP3 inflammasome assembly is regulated by phosphorylation of the pyrin domain. J Exp Med (2017) 214:1725-36. doi:10.1084/jem.20160933

29. Song N, Liu Z, Xue W, Bai Z, Wang Q, Dai J, et al. NLRP3 phosphorylation is an essential priming event for inflammasome activation. Mol Cell (2017) 68(1):185-97.e6. doi:10.1016/j.molcel.2017.08.017
30. Zhang Z, Meszaros G, He WT, Xu Y, de Fatima Magliarelli H, Mailly L, et al. Protein kinase $\mathrm{D}$ at the Golgi controls NLRP3 inflammasome activation. J Exp Med (2017) 214:2671-93. doi:10.1084/jem.20162040

31. Diaz-Gallo L, Espino-Paisán L, Fransen K, Gómez-García M, van Sommeren S, Cardeña C, et al. Differential association of two PTPN22 coding variants with Crohn's disease and ulcerative colitis. Inflamm Bowel Dis (2011) 17: 2287-94. doi:10.1002/ibd.21630

32. Begovich AB, Carlton VE, Honigberg LA, Schrodi SJ, Chokkalingam AP, Alexander $\mathrm{HC}$, et al. A missense single-nucleotide polymorphism in a gene encoding a protein tyrosine phosphatase (PTPN22) is associated with rheumatoid arthritis. Am J Hum Genet (2004) 75:330-7. doi:10.1086/422827

33. Kyogoku C, Ortmann WA, Lee A, Selby S, Carlton VE, Chang M, et al. Genetic association of the R620W polymorphism of protein tyrosine phosphatase PTPN22 with human SLE. Am J Hum Genet (2004) 75:504-7. doi:10.1086/423790

34. Bottini N, Musumeci L, Alonso A, Rahmouni S, Nika K, Rostamkhani M, et al. A functional variant of lymphoid tyrosine phosphatase is associated with type I diabetes. Nat Genet (2004) 36:337-8. doi:10.1038/ng1323

35. Tasken K, Aandahl EM. Localized effects of cAMP mediated by distinct routes of protein kinase A. Physiol Rev (2004) 84:137-67. doi:10.1152/ physrev.00021.2003

36. Sokolowska M, Chen LY, Liu Y, Martinez-Anton A, Qi HY, Logun C, et al. Prostaglandin E2 inhibits NLRP3 inflammasome activation through EP4 receptor and intracellular cyclic AMP in human macrophages. J Immunol (2015) 194:5472-87. doi:10.4049/jimmunol.1401343

37. Martin BN, Wang C, Willette-Brown J, Herjan T, Gulen MF, Zhou H, et al. IKKalpha negatively regulates ASC-dependent inflammasome activation. Nat Commun (2014) 5:4977. doi:10.1038/ncomms5977

38. Zhou R, Yazdi AS, Menu P, Tschopp J. A role for mitochondria in NLRP3 inflammasome activation. Nature (2011) 469:221. doi:10.1038/ nature09663

39. Yang C, Kim J, Kim TS, Lee PY, Kim SY, Lee H, et al. Small heterodimer partner interacts with NLRP3 and negatively regulates activation of the NLRP3 inflammasome. Nat Commun (2015) 6:6115. doi:10.1038/ ncomms7115

40. Py BF, Kim M, Vakifahmetoglu-Norberg H, Yuan J. Deubiquitination of NLRP3 by BRCC3 critically regulates inflammasome activity. Mol Cell (2013) 49:331-8. doi:10.1016/j.molcel.2012.11.009

41. Kawashima A, Karasawa T, Tago K, Kimura H, Kamata R, Usui-Kawanishi F, et al. ARIH2 ubiquitinates NLRP3 and negatively regulates NLRP3 inflammasome activation in macrophages. J Immunol (2017) 199:3614-22. doi:10.4049/ jimmunol.1700184

42. Han S, Lear TB, Jerome JA, Rajbhandari S, Snavely CA, Gulick DL, et al. Lipopolysaccharide primes the NALP3 inflammasome by inhibiting its ubiquitination and degradation mediated by the SCFFBXL2 E3 ligase. J Biol Chem (2015) 290:18124-33. doi:10.1074/jbc.M115.645549

43. Song H, Liu B, Huai W, Yu Z, Wang W, Zhao J, et al. The E3 ubiquitin ligase TRIM31 attenuates NLRP3 inflammasome activation by promoting proteasomal degradation of NLRP3. Nat Commun (2016) 7:13727. doi:10.1038/ ncomms 13727

44. Yan Y, Jiang W, Liu L, Wang X, Ding C, Tian Z, et al. Dopamine controls systemic inflammation through inhibition of NLRP3 inflammasome. Cell (2015) 160:62-73. doi:10.1016/j.cell.2014.11.047

45. Lopez-Castejon G, Luheshi NM, Compan V, High S, Whitehead RC, Flitsch S, et al. Deubiquitinases regulate the activity of caspase-1 and interleukin-1beta secretion via assembly of the inflammasome. J Biol Chem (2013) 288:2721-33. doi:10.1074/jbc.M112.422238

46. Juliana C, Fernandes-Alnemri T, Kang S, Farias A, Qin F, Alnemri ES. Non-transcriptional priming and deubiquitination regulate NLRP3 inflammasome activation. JBiol Chem (2012) 287:36617-22. doi:10.1074/jbc. M112.407130

47. Basu S, Nagy JA, Pal S, Vasile E, Eckelhoefer IA, Bliss VS, et al. The neurotransmitter dopamine inhibits angiogenesis induced by vascular permeability factor/vascular endothelial growth factor. Nat Med (2001) 7:569-74. doi: $10.1038 / 87895$

48. Chakroborty D, Chowdhury UR, Sarkar C, Baral R, Dasgupta PS, Basu S. Dopamine regulates endothelial progenitor cell mobilization from mouse bone marrow in tumor vascularization. J Clin Invest (2008) 118:1380-9. doi:10.1172/JCI33125 
49. Juliana C, Fernandes-Alnemri T, Wu J, Datta P, Solorzano L, Yu JW, et al. Anti-inflammatory compounds parthenolide and Bay 11-7082 are direct inhibitors of the inflammasome. J Biol Chem (2010) 285:9792-802. doi:10.1074/jbc. M109.082305

50. He Y, Varadarajan S, Munoz-Planillo R, Burberry A, Nakamura Y, Nunez G. 3,4-methylenedioxy-beta-nitrostyrene inhibits NLRP3 inflammasome activation by blocking assembly of the inflammasome. J Biol Chem (2014) 289: 1142-50. doi:10.1074/jbc.M113.515080

51. Cocco M, Miglio G, Giorgis M, Garella D, Marini E, Costale A, et al. Design, synthesis, and evaluation of acrylamide derivatives as direct NLRP3 inflammasome inhibitors. ChemMedChem (2016) 11:1790-803. doi:10.1002/ cmdc. 201600055

52. Duncan JA, Bergstralh DT, Wang Y, Willingham SB, Ye Z, Zimmermann AG, et al. Cryopyrin/NALP3 binds ATP/dATP, is an ATPase, and requires ATP binding to mediate inflammatory signaling. Proc Natl Acad Sci U S A (2007) 104:8041-6. doi:10.1073/pnas.0611496104

53. Shim D, Shin W, Yu S, Kim B, Ye S, Koppula S, et al. BOT-4-one attenuates NLRP3 inflammasome activation: NLRP3 alkylation leading to the regulation of its ATPase activity and ubiquitination. Sci Rep (2017) 7:15020. doi:10.1038/ s41598-017-15314-8

54. Mishra BB, Rathinam VA, Martens GW, Martinot AJ, Kornfeld H, Fitzgerald KA, et al. Nitric oxide controls the immunopathology of tuberculosis by inhibiting NLRP3 inflammasome-dependent processing of IL-1 [beta]. Nat Immunol (2013) 14:52-60. doi:10.1038/ni.2474

Conflict of Interest Statement: The authors declare that the research was conducted in the absence of any commercial or financial relationships that could be construed as a potential conflict of interest.

Copyright $\odot 2018$ Shim and Lee. This is an open-access article distributed under the terms of the Creative Commons Attribution License (CC BY). The use, distribution or reproduction in other forums is permitted, provided the original author(s) and the copyright owner are credited and that the original publication in this journal is cited, in accordance with accepted academic practice. No use, distribution or reproduction is permitted which does not comply with these terms. 